\title{
PERDAS DE SOLO E NUTRIENTES POR EROSÃO NUM ARGISSOLO COM RESÍDUOS VEGETAIS DE CANA-DE-AÇÚCAR ${ }^{1}$
}

\author{
MARCÍLIO V. MARTINS FILHO ${ }^{2}$, THIAGO T. LICCIOTI ${ }^{3}$, GENER T. PEREIRA ${ }^{4}$, \\ JOSÉ MARQUES JÚNIOR ${ }^{5}$, RODRIGO B. SANCHEZ
}

RESUMO: O presente trabalho teve como objetivo determinar as perdas de solo, matéria orgânica (MO) e nutrientes (P, K, Ca e Mg) por erosão, em área cultivada com cana-de-açúcar, cuja palhada é mantida sobre a superfície do solo, localizada em Catanduva - SP, sob um Argissolo Vermelho-Amarelo. Em parcelas experimentais, com $0\left(\mathrm{CS}_{0}\right), 50\left(\mathrm{CS}_{50}\right)$ e $100 \%\left(\mathrm{CS}_{100}\right)$ de cobertura sobre a superfície do solo, aplicou-se chuva simulada com intensidade de $60 \mathrm{~mm} \mathrm{~h}^{-1}$, durante 65 minutos. Análises do sedimento erodido indicaram taxas de enriquecimento da seguinte ordem: 2,7 a 1,9 (MO), 3,8 a 2,7 (P), 1,3 a 1,7 (K), 3,9 a 3,6 (Ca) e 2,9 a 2,6 (Mg) vezes em relação ao solo original para $\mathrm{CS}_{0}$ e $\mathrm{CS}_{50}$, respectivamente. $\mathrm{A} \mathrm{CS}_{50}$ propiciou controle significativo da erosão de $69 \%$, mas não reduziu a concentração de $\mathrm{MO}$ e nutrientes no sedimento erodido. A $\mathrm{CS}_{100}$ foi significativamente eficiente no controle da erosão (89\%) e na redução das concentrações de MO (69\%), P (88\%), K (23\%), Ca (74\%) e Mg (75\%) no sedimento.

PALAVRAS-CHAVE: processo erosivo, simulação de chuva, cobertura do solo.

\section{SOIL AND NUTRIENTS LOSSES OF AN ALFISOL WITH SUGARCANE CROP RESIDUE}

\begin{abstract}
The objective of this study was to determine the losses of soil, organic matter (OM) and nutrients $(\mathrm{P}, \mathrm{K}, \mathrm{Ca}$, and $\mathrm{Mg}$ ) caused by erosion in an area cultivated with sugarcane containing controlled amount of crop residues in soil surface, in Catanduva city, São Paulo State, Brazil. The soil at the experimental site is an Alfisol. Test plots were $0\left(\mathrm{CS}_{0}\right), 50\left(\mathrm{CS}_{50}\right)$ and $100 \%\left(\mathrm{CS}_{100}\right)$ of crop residues cover, submitted to simulated rainfall simulated of $60 \mathrm{~mm} \mathrm{~h}^{-1}$, during 65 minutes. Analyses of the eroded sediment indicate an enrichment ratio of 2.7 - $1.9(\mathrm{OM}), 3.8$ - $2.7(\mathrm{P}), 1.3$ $1.7(\mathrm{~K}), 3.9-3.6(\mathrm{Ca})$, and $2.9-2.6(\mathrm{Mg})$ times in relation to the $\mathrm{CS}_{0}$ and $\mathrm{CS}_{50}$, respectively. The $\mathrm{CS}_{50}$ was more efficient for controlling the erosion processes in $69 \%$, but not for the reduction of the $\mathrm{OM}$ and nutrients losses in the eroded sediment. On the other hand, $\mathrm{CS}_{100}$ was efficient for erosion control of $89 \%$ and in the reduction of OM (69\%), P (88\%), K (23\%), Ca (74\%) and $\mathrm{Mg}$ $(75 \%)$ in the eroded sediment.
\end{abstract}

KEYWORDS: erosion processes, rainfall simulation, soil cover.

\section{INTRODUÇÃO}

A colheita mecanizada vem ganhando espaço no setor canavieiro com o benefício de promover a cobertura do solo com resíduos da cultura de cana-de-açúcar. Tal sistema de manejo é importante principalmente em solos suscetíveis à erosão, a exemplo dos Argissolos, que ocorrem em extensas áreas do Estado de São Paulo. Com a manutenção dos resíduos vegetais na superfície do solo, esses poderão interceptar as gotas de chuva e dissipar a sua energia, evitando a desagregação das partículas e a formação do selamento superficial. Desse modo, a capacidade de

\footnotetext{
${ }^{1}$ Extraído do Trabalho de Iniciação Científica do segundo autor para Graduação em Agronomia. Projeto financiado pela UNESP e Usina São Domingos Açúcar e Álcool S.A.

${ }^{2}$ Professor Adjunto, Departamento de Solos e Adubos, UNESP, Via de Acesso Prof. Paulo Donato Castellane, km 5, Jaboticabal SP, mfilho@fcav.unesp.br

${ }^{3}$ Eng $^{\mathrm{o}}$. Agrônomo, AGRICOOP, thiagoliccioti@yahoo.com.br

${ }^{4}$ Prof. Doutor, UNESP, Departamento de Solos e Adubos, marques@fcav.unesp.br

${ }^{5}$ Prof. Doutor, UNESP, Departamento de Ciências Exatas, genertp@ fcav.unesp.br

${ }^{6}$ Doutor em Agronomia, Usina São Domingos Açúcar e Álcool S.A. rodrigo@usinasaodomingos.com.br

Recebido pelo Conselho Editorial em: 17-3-2008
}

Aprovado pelo Conselho Editorial em: 18-2-2009

Eng. Agríc., Jaboticabal, v.29, n.1, p.8-18, jan./mar. 2009 
infiltração e a estabilidade estrutural, características físicas mais expressivas e intimamente relacionadas com a erodibilidade (BRADY \& WEIL, 2002), seriam pouco afetadas, contribuindo para a redução da velocidade da enxurrada e, consequentemente, a redução na sua capacidade de desagregação e de transporte das partículas do solo. Contudo, há poucas pesquisas direcionadas a comprovar e entender como tal sistema de manejo atua no controle das perdas de solo, matéria orgânica e nutrientes, associados ao sedimento erodido.

É conhecido que sistemas de cultivo, como o plantio direto e o mínimo, reduzem as perdas por erosão, em comparação ao sistema convencional (HERNANI et al.,1999; BERTOL et al., 2004; BERTOL et al., 2007). IZIDORIO et al. (2005) constataram, em sistema de cultivo da cana-de-açúcar, com a queima da palhada antes da colheita, que as perdas de nutrientes por erosão são expressivas com o sedimento, o qual apresentou altas taxas de enriquecimento: 1,62 (matéria orgânica, MO); 4,30 (P); 1,17 (K); 1,33 (Ca) e 1,24 (Mg) vezes em relação ao solo original.

O cultivo da cana-de-açúcar em São Paulo é de suma importância para a sua economia (IZIDORIO, 2004). Logo, a colheita da cana-de-açúcar sem a despalha, mantendo os resíduos vegetais sobre a superfície do solo, torna-se um sistema interessante sob o ponto de vista da conservação do solo e da água. BEZERRA \& CANTALICE (2006) verificaram que a presença de resíduos na superfície diminui o impacto das gotas da chuva e o escoamento superficial, o que reduz a desagregação das partículas do solo e controla a erosão.

BRAIDA \& CASSOL (1999) observaram que os resíduos vegetais, quando mantidos sobre a superfície do solo, têm alta eficiência no controle da erosão. Porém, essa eficiência está ligada ao tipo e à quantidade de resíduos vegetais utilizados para tal controle. Verificaram, ainda, que as mesmas quantidades de palha de milho e trigo, apesar de os resíduos de milho não cobrirem o solo tão eficientemente quanto os do trigo, são igualmente eficientes em reduzir a erosão, devido à similaridade dos seus efeitos sobre o fluxo superficial.

$\mathrm{O}$ efeito da cobertura vegetal do solo sobre a erosão pode ser concebido como: Tipo I $\left(\mathrm{Ci}_{\mathrm{I}}\right)$, relativo à cobertura vegetal oferecida pelo dossel; tipo II $\left(\mathrm{Ci}_{\mathrm{II}}\right)$, relativo à cobertura vegetal em contato direto com a superfície do solo, e tipo III ( $\mathrm{Ci}_{\text {III }}$ ), efeito da incorporação de resíduos vegetais ao solo em função do seu uso e manejo (FOSTER, 1982). O efeito tipo I é aquele devido à interceptação das gotas da água da chuva pelo dossel ou parte aérea das culturas, o qual é dependente da altura e da densidade do dossel (LAFLEN et al.,1985). A cobertura em contato direto com a superfície do solo, efeito tipo II, permite que a energia cinética das gotas da chuva seja dissipada junto à superfície, além de ser obstáculo à ação cisalhante da enxurrada (FOSTER, 1982; BRAIDA \& CASSOL, 1999). Segundo WISCHMEIER (1975), o efeito tipo III é aquele resultante dos resíduos incorporados e do efeito residual do uso e do manejo do solo na desagregação e no transporte de sedimentos erodidos. BRAIDA \& CASSOL (1999) afirmaram que o efeito II possibilita maior controle do processo de erosão do que o efeito I.

SCHAEFER et al. (2002) determinaram perdas de nutrientes por erosão, sob condições de chuva simulada, em diferentes percentagens de cobertura da superfície do solo proporcionadas por manta sintética $(0 ; 20 ; 40 ; 60 ; 80$ e 100\%), num Argissolo Vermelho-Amarelo. Em todas as percentagens de cobertura, a tendência de perdas de nutrientes no sedimento erodido foi $\mathrm{Ca}>\mathrm{Mg}>$ $\mathrm{K}>\mathrm{P}$. Verificaram, ainda, a existência de coeficientes de correlação positivos e significativos entre as perdas de $\mathrm{P}, \mathrm{K}, \mathrm{Ca}$ e $\mathrm{Mg}$ com as de matéria orgânica.

SILVA et al. (2005) obtiveram perdas de nutrientes com o sedimento erodido, em Latossolo Vermelho distroférrico (LVdf) e Cambissolo Háplico Tb distrófico (CXbd) desprovidos de vegetação e preparados no sentido do declive, com a seguinte tendência: $\operatorname{LVdf}\left(0,12 \mathrm{~m} \mathrm{~m}^{-1}\right)$ - Ca $\left(3,88 \mathrm{~kg} \mathrm{ha}^{-1}\right)>\mathrm{K}\left(2,34 \mathrm{~kg} \mathrm{ha}^{-1}\right)>\operatorname{Mg}\left(0,51 \mathrm{~kg} \mathrm{ha}^{-1}\right)>\mathrm{P}\left(0,23 \mathrm{~kg} \mathrm{ha}^{-1}\right)$ e CXbd $\left(0,15 \mathrm{~m} \mathrm{~m}^{-1}\right)-\mathrm{K}$ $\left(26,85 \mathrm{~kg} \mathrm{ha}^{-1}\right)>\mathrm{Ca}\left(25,03 \mathrm{~kg} \mathrm{ha}^{-1}\right)>\mathrm{P}\left(3,89 \mathrm{~kg} \mathrm{ha}^{-1}\right)>\operatorname{Mg}\left(3,74 \mathrm{~kg} \mathrm{ha}^{-1}\right)$.

IZIDORIO et al. (2005) avaliaram as perdas de nutrientes no sedimento erodido oriundo de área de cana-de-açúcar submetida à queima, sob chuva artificial, num Latossolo Vermelho 
eutroférrico desprovido de vegetação e preparado no sentido do declive $\left(0,08 \mathrm{~m} \mathrm{~m}^{-1}\right)$. Obtiveram perdas de P, K, Ca e Mg de 1,07 kg ha ${ }^{-1}, 1,59 \mathrm{~kg} \mathrm{ha}^{-1}, 10,24 \mathrm{~kg} \mathrm{ha}^{-1}$ e 1,91 $\mathrm{kg} \mathrm{ha}^{-1}$, respectivamente.

Diversos trabalhos abordam as perdas de nutrientes por erosão, para diferentes sistemas de manejo e cobertura do solo, principalmente para culturas anuais (HERNANI et al., 1999; BERTOL et al., 2004; BERTOL et al., 2007). Contudo, para culturas como a da cana-de-açúcar, sob colheita mecanizada, há carência de informações na literatura sobre as perdas de nutrientes por erosão.

Para avaliar a eficiência da cobertura superficial de um Argissolo com resíduos da cultura de cana-de-açúcar, no controle das perdas de solo, matéria orgânica e nutrientes por erosão, foi realizado este trabalho na região de Catanduva - SP, em área com uso de colheita mecânica.

\section{MATERIAL E MÉTODOS}

A área na qual foi conduzido o presente trabalho, localiza-se no município de Catanduva, noroeste do Estado de São Paulo, cujas coordenadas geográficas são: latitude $21^{\circ} 05^{\prime} 57,11^{\prime \prime}$ e longitude $49^{\circ} 01^{\prime} 02,08^{\prime \prime}$ W. O clima, segundo a classificação de Köeppen, é denominado tropical quente úmido, tipo Aw, seco no inverno, com precipitação média anual de $1.350 \mathrm{~mm}$. Ressalta-se que a área apresenta histórico de mais de 20 anos consecutivos com cultivo de cana-de-açúcar e 5 anos de colheita mecanizada. Desse modo, a cobertura vegetal da área, na época da realização deste trabalho, era constituída por resíduos de cana-de-açúcar, os quais foram mantidos sobre a superfície após a colheita mecânica da cultura.

O solo foi classificado como Argissolo Vermelho-Amarelo eutrófico, A moderado (EMBRAPA, 1999), cujos valores dos principais atributos químicos e físicos são apresentados nas Tabelas 1 e 2.

TABELA 1. Atributos químicos do solo na profundidade 0,00 a $0,20 \mathrm{~m}$. Soil chemical attributes in $0,00-0,20 \mathrm{~m}$ depth.

\begin{tabular}{ccccccccc}
\hline $\mathrm{pH}$ & $\begin{array}{c}\mathrm{MO} \\
\mathrm{g} \mathrm{dm}^{-3}\end{array}$ & $\begin{array}{c}\mathrm{P} \\
\mathrm{mg} \mathrm{dm}^{-3}\end{array}$ & $\mathrm{~K}$ & $\mathrm{Ca}$ & $\begin{array}{c}\mathrm{Mg} \\
\mathrm{mmol}_{\mathrm{c}} \mathrm{dm}^{-3}\end{array}$ & $\mathrm{SB}$ & $\mathrm{CTC}$ & $\begin{array}{c}\mathrm{V} \\
\%\end{array}$ \\
\hline 5,0 & 16 & 34 & 2 & 20 & 9 & 31 & 48,9 & 63,4 \\
\hline
\end{tabular}

TABELA 2. Atributos físicos do solo na profundidade 0,00 a $0,20 \mathrm{~m}$. Physical soil attributes in 0,00 - $0,20 \mathrm{~m}$ depth.

\begin{tabular}{ccccccc}
\hline Argila & $\begin{array}{c}\text { Silte } \\
\mathrm{g} \mathrm{kg}^{-1}\end{array}$ & Areia & Ma & $\begin{array}{c}\text { Mi } \\
\text {------------- }\end{array}$ & VTP & $\begin{array}{c}\mathrm{ds} \\
\mathrm{kg} \mathrm{dm}^{-3}\end{array}$ \\
\hline 180 & 31 & 789 & 16,36 & 20,16 & 36,52 & 1,41 \\
\hline
\end{tabular}

Ma - macroporosidade; Mi - microporosidade; VTP - volume total de poros; ds - densidade do solo.

A quantificação das perdas de solo e de água por erosão, sob condições de chuva artificial, foi realizada sob três condições de cobertura vegetal em contacto direto com a superfície: 1) ausência de cobertura $\left(\mathrm{CS}_{0}\right)$; 2) $50 \%$ de cobertura $\left(\mathrm{C}_{50}\right)$; e 3) $100 \%$ de cobertura $\left(\mathrm{C}_{100}\right)$. As percentagens de cobertura foram avaliadas segundo método da linha de transeção descrito por SHELTON et al. (1993). Uma moldura com 55 células, cada uma com espaçamento de $1,0 \mathrm{~m}$ entre linhas e $0,75 \mathrm{~m}$ entre colunas, foi utilizada para a composição da cobertura de $50 \%$ com o preenchimento aleatório de 27,5 malhas com o resíduo. A dose de aplicação de resíduos foi a mesma para $\mathrm{C}_{50}$ e $\mathrm{C}_{100}$ e igual a $1,4 \mathrm{~kg} \mathrm{~m}^{-2}$. As referidas percentagens constituíram-se nos tratamentos aplicados num delineamento experimental, em blocos inteiramente casualizados (DBC). Foram realizadas nove repetições de cada tratamento, totalizando 27 parcelas experimentais.

Parcelas com 3,50 m de largura e 11,00 m de comprimento $\left(38,5 \mathrm{~m}^{2}\right)$ foram delimitadas com chapas metálicas nas laterais e na parte superior, e por calhas coletoras metálicas em seus limites inferiores. As calhas coletoras com 3,50 m de largura convergiam para a saída de $0,065 \mathrm{~m}$ de diâmetro. Amostras de enxurrada foram coletadas nessas calhas para quantificar as perdas de solo e 
de água. Todas as parcelas foram alinhadas no sentido da declividade do terreno, a qual, em média, foi de $0,052 \mathrm{~m} \mathrm{~m}^{-1}$. O preparo do solo também foi realizado no sentido do declive.

As parcelas experimentais foram submetidas a chuvas simuladas com intensidade média de $60 \mathrm{~mm} \mathrm{~h}^{-1}$ (I), durante 65 minutos. Utilizou-se, para realizar as referidas precipitações, de simulador de chuva de hastes rotativas do tipo Swanson, com bicos veejet 80-100, previamente calibrado e nivelado no terreno, como descrito por MARTINS FILHO et al. (2004).

Trinta e seis pluviômetros, alinhados no sentido do declive, na área de ação do simulador de chuvas, como descrito por MARTINS FILHO (1999), foram utilizados para determinar as intensidades das precipitações produzidas pelo simulador, nas áreas ocupadas pelas parcelas experimentais.

Amostragens para medidas de vazões dos escoamentos superficiais e das concentrações de sedimentos foram realizadas no quinto minuto após o início da enxurrada e, a partir daí, a cada cinco minutos, como o realizado por MARTINS FILHO et al. (2004). Amostras foram coletadas em recipientes de vidro com capacidade de $1 \mathrm{~L}$, cronometrando-se o tempo de coleta. Logo após as coletas, os recipientes foram fechados e conduzidos ao laboratório para quantificação da concentração de sedimentos e volume de solução e, consequentemente, determinação das taxas de perdas de solo, água $(\mathrm{Q})$ e nutrientes no sedimento.

Os volumes de solução coletados foram avaliados gravimetricamente, em balança com resolução de $0,01 \mathrm{~g}$ e, em seguida, as amostras foram deixadas em repouso por $24 \mathrm{~h}$ para a deposição de sedimentos. Após o período de repouso de $24 \mathrm{~h}$, as amostras foram levadas à estufa a $105^{\circ} \mathrm{C}$ até secagem completa. Após secagem, as amostras foram pesadas, determinando-se o peso de sedimento de cada uma. A concentração de sedimentos foi obtida considerando-se o volume da solução, a densidade da água e do sedimento na solução (MARTINS FILHO et al., 2004). Desse modo, determinaram-se para cada parcela e tratamento as perdas totais por erosão hídrica (A) durante cada uma das chuvas simuladas. Taxas de infiltração de água no solo foram determinadas pela diferença entre I e Q.

A erosividade, fator $\mathrm{R}$, para as chuvas simuladas, foi obtida como descrito por MARTINS FILHO (1987). O fator relevo (LS) foi determinado como proposto por WISCHMEIER \& SMITH (1978) para cada parcela experimental.

As perdas de solo (A) obtidas nas parcelas experimentais foram utilizadas na determinação da erodibilidade do solo (fator $\mathrm{K}$ ) e do subfator $\mathrm{Ci}_{\text {II }}$ como:

$$
\begin{aligned}
& \mathrm{K}=\frac{\mathrm{A}}{(\mathrm{R} \mathrm{LS})_{\mathrm{Cs}_{0}}} \\
& \mathrm{Ci}_{\mathrm{II}}=\frac{[\mathrm{A} /(\mathrm{R} \mathrm{LS})]_{\mathrm{CS} \mathrm{i}}}{[\mathrm{A} /(\mathrm{R} \mathrm{LS})]_{\mathrm{CS} 0}}
\end{aligned}
$$

em que,

$\mathrm{K}$ - erodibilidade do solo, $\mathrm{Mg}$ ha $\mathrm{h} \mathrm{ha}^{-1} \mathrm{MJ}^{-1} \mathrm{~mm}^{-1}$;

A / R LS - taxa de erosão pelo produto da erosividade (R) e fator relevo (LS);

$\mathrm{CS}_{\mathrm{i}}$ - tratamentos com $50 \%$ e $100 \%$ de cobertura residual, e

$\mathrm{CS}_{0}$ - tratamento sem cobertura residual.

Para a condição de ausência de vegetação, o subfator $\left(\mathrm{Ci}_{\mathrm{III}}\right)$ assume valor unitário.

Nas análises químicas do solo e do sedimento erodido, $\mathrm{Ca}, \mathrm{Mg}, \mathrm{K}$ e $\mathrm{P}$ foram extraídos pelo método da resina trocadora de íons (RAIJ et al., 1987). O carbono orgânico foi determinado seguindo a metodologia da EMBRAPA (1997). O pH foi determinado potenciometricamente em solução de $\mathrm{CaCl}_{2}$ 0,01 M. 
Os dados foram submetidos à análise da variância, segundo o $\mathrm{DBC}$, sendo utilizado o teste de Tukey, a 5\%, para as comparações múltiplas das médias. Diferenças com nível mínimo de significância inferior a 5\% foram consideradas significativas. Análises de regressão também foram conduzidas entre taxa de infiltração de água (i) e tempo de duração (t), e perdas de solo (A), e percentagem de cobertura por resíduos (CS). Ajustou-se para i em função de $t$ o modelo de Kostiakov $\left(\mathrm{i}=\mathrm{a} \mathrm{t}^{-\mathrm{k}}\right)$, e para A em função de CS, modelo exponencial $\left(\mathrm{A}=\mathrm{z} \mathrm{e}^{-\mathrm{b} C S}\right)$, tal que $\mathrm{a}, \mathrm{k}, \mathrm{z}$ e $\mathrm{b}$ são constantes ajustadas. As análises estatísticas foram realizadas com o programa Statistica (STATSOFT, 1994).

\section{RESULTADOS E DISCUSSÃO}

Os efeitos da cobertura do solo na taxa média de infiltração de água (i), para as parcelas com 0\%, 50\% e $100 \%$ de resíduos de palha de cana-de-açúcar em contato direto com a superfície do Argissolo, estão representados na Figura 1. Esses resultados corroboram BRAIDA \& CASSOL (1999), MARTINS FILHO et al. (2004) e BEZERRA \& CANTALICE (2006), que verificaram que o aumento na quantidade de palha sobre o solo resultou em maior proteção contra o impacto direto das gotas da chuva. Como consequência dessa proteção, há menor selamento superficial e maior infiltração da água no solo. Além disso, os resíduos na superfície do solo reduzem a velocidade do escoamento superficial e aumentam a resistência e a altura mínima da lâmina para que ocorra escoamento (BRAIDA \& CASSOL, 1999; CASSOL et al., 2004), o que favorece a infiltração de água.

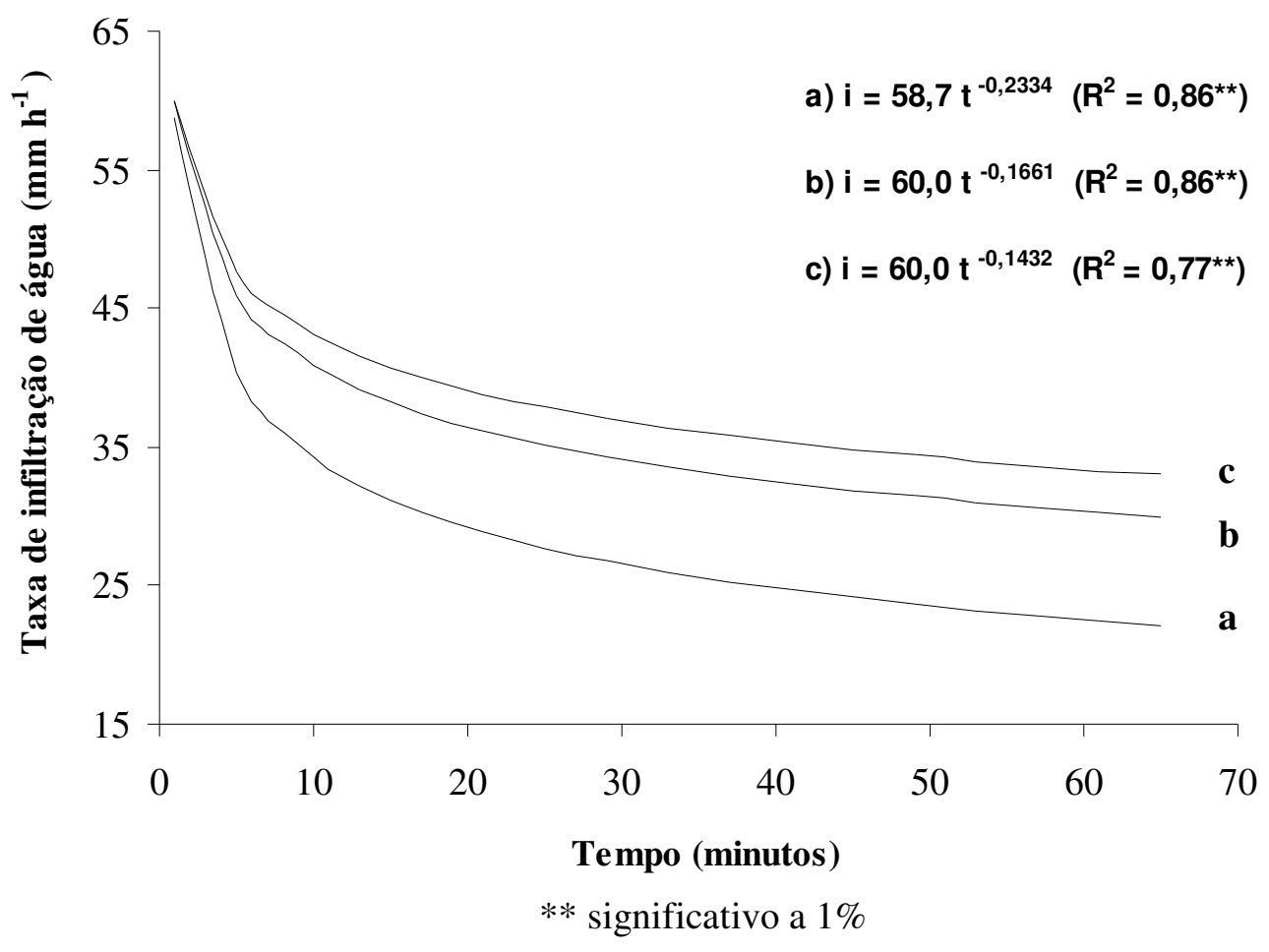

FIGURA 1. Taxa de infiltração de água no Argissolo: a) $0 \%$; b) $50 \%$, e c) $100 \%$ de palha de cana-de-açúcar em contato direto com a superfície do solo. Infiltration rate in the Alfisol with sugarcane crop residue: a) $0 \%$; b) $50 \%$ e c) $100 \%$.

A erosão (A) decresceu exponencialmente com o aumento da percentagem de cobertura oferecida ao solo (CS) pela palha da cana-de-açúcar (Figura 2). Tal aspecto reforça o efeito benéfico que a palhada tem no controle da erosão do solo, o que também foi verificado por BRAIDA \& CASSOL (1999), CASSOL \& LIMA (2003) e CASSOL et al. (2004). 


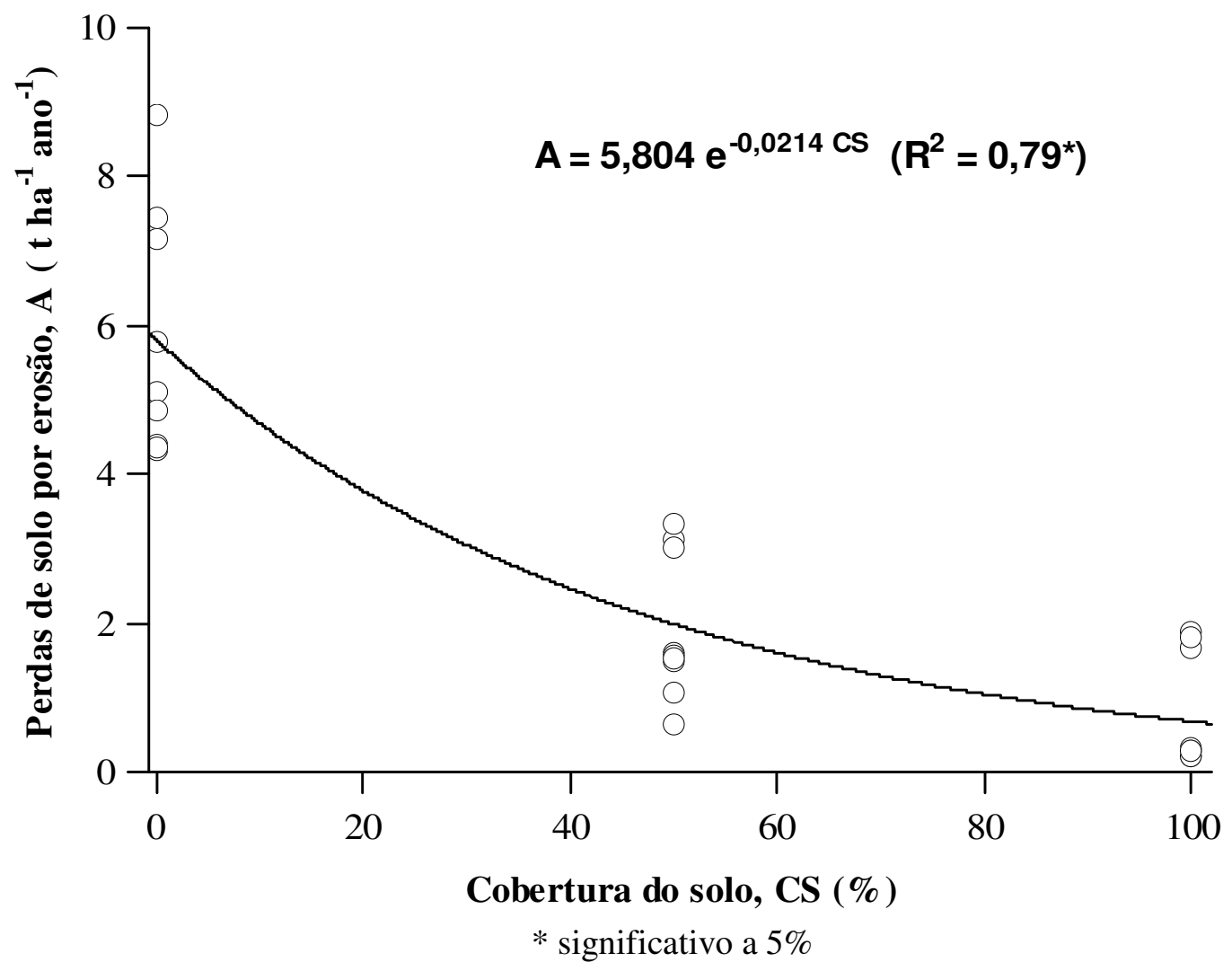

FIGURA 2. Perdas de solo por erosão em função da percentagem de cobertura da superfície do solo por palha de cana-de-açúcar. Relation between soil loss (A) and sugarcane crop residue cover (CS).

As diferenças significativas dos valores médios de perdas por erosão e do subfator $\mathrm{Ci}_{\text {II }}$, para cada nível de cobertura por palha sobre a superfície do solo, são apresentadas na Tabela 3. Verificou-se decréscimo significativo das perdas de solo por erosão, quando a percentagem de cobertura por palha de cana-de-açúcar foi superior a 50\% (Tabela 3). Isso ocorreu em consequência da redução da desagregação do solo pelo impacto direto das gotas da chuva. Com redução da área exposta ao impacto de $50 \%$ e considerando-se o subfator $\mathrm{Ci}_{\text {II }}$ igual a 0,32 , pode-se dizer que a estimativa da redução da erosão foi de 68\%. Desse modo, $18 \%$ (68\%-50\%) das estimativas da redução na erosão foram devidas ao efeito da palha sobre o transporte de sedimentos pelo fluxo laminar e, provavelmente, também pela diminuição na taxa de desagregação pelo aumento da profundidade do fluxo. No caso de $100 \%$ de cobertura do solo, o subfator $\mathrm{Ci}_{\text {II }}$ igual a 0,11 corresponde à estimativa de redução média de $89 \%$ na taxa de erosão. Logo, $11 \%$ (100\% - 89\%) das estimativas de redução da erosão podem ter ocorrido pelo controle do escoamento e/ou pelo aumento da profundidade do fluxo, a exemplo do verificado por BRAIDA \& CASSOL (1999) para palhas de milho e trigo mantidas em contato direto com a superfície do solo.

As parcelas com $100 \%$ de cobertura apresentaram subfator $\mathrm{Ci}_{\text {II }}$ médio igual a 0,11 , o que demonstra que, mesmo nessa condição, ocorreu erosão (Tabela 3). Tal ocorrência não é a esperada, visto que havia $100 \%$ de cobertura da superfície do solo. Nessa condição, não há desagregação pelo impacto das gotas da chuva. Contudo, o Argissolo da área apresenta agregação muito fraca superficialmente. Portanto, as operações de preparo do solo, efetuadas antes das chuvas simuladas, contribuíram para a desagregação e o transporte das suas partículas pelo fluxo superficial existente sob os resíduos vegetais, o que resultou numa taxa de erosão ainda mensurável como o obtido por BRAIDA \& CASSOL (1999). Ressalta-se aqui que BRAIDA \& CASSOL (1999) realizaram o preparo em sentido transversal ao declive. 
TABELA 3. Perdas de solo por erosão (A) e subfator cobertura e manejo $\left(\mathrm{Ci}_{\mathrm{II}}\right)$ em função da cobertura do solo (CS) por palha de cana-de-açúcar. Soil loss (A) and covermanagement subfactor $\left(\mathrm{Ci}_{\mathrm{II}}\right)$, when the soil should have $0 \%$ to $100 \%$ of its surface covered by sugarcane crop residues (CS).

\begin{tabular}{ccc}
\hline Cobertura do Solo CS $(\%)$ & $\mathrm{A}\left(\mathrm{t} \mathrm{ha}^{-1} \mathrm{ano}^{-1}\right)$ & Subfator $\mathrm{i}_{\text {II }}$ \\
\hline 0 & $5,81 \mathrm{a}$ & $1,00 \mathrm{a}$ \\
50 & $1,93 \mathrm{~b}$ & $0,32 \mathrm{~b}$ \\
100 & $0,77 \mathrm{~b}$ & $0,11 \mathrm{c}$ \\
\hline C.V.(\%) & 42 & 16 \\
\hline
\end{tabular}

Médias seguidas de mesma letra na coluna não diferem significativamente, pelo teste Tukey, a 5\% de probabilidade.

As concentrações médias de $\mathrm{MO}, \mathrm{P}, \mathrm{K}, \mathrm{Ca}$ e $\mathrm{Mg}$ foram maiores nas parcelas em que a cobertura vegetal foi inferior a 50\% (Tabela 4). As concentrações de $\mathrm{MO}, \mathrm{P}, \mathrm{K}, \mathrm{Ca}$ e $\mathrm{Mg}$ não apresentaram diferença significativa $(\mathrm{p}>0,05)$ entre as parcelas com $0 \%$ e $50 \%$ de cobertura do solo $\left(\mathrm{CS}_{0}\right.$ e $\mathrm{CS}_{50}$, respectivamente). Porém, parcelas com $100 \%$ de cobertura $\left(\mathrm{CS}_{100}\right)$ apresentaram diferença significativa $(\mathrm{p}<0,05)$ em relação a $\mathrm{CS}_{0}$ e a $\mathrm{CS}_{50}$, para as concentrações de $\mathrm{MO}, \mathrm{P}, \mathrm{Ca}$ e $\mathrm{Mg}$ no sedimento erodido. Isso sugere que a $\mathrm{CS}_{50}$ não confere redução significativa na concentração de $\mathrm{MO}$ e nutrientes $\left(\mathrm{P}, \mathrm{Ca}\right.$ e $\mathrm{Mg}$ ) no sedimento erodido. Entretanto, a $\mathrm{CS}_{50}$ propicia controle significativo $(\mathrm{p}<0,005)$ da erosão em relação a $\mathrm{CS}_{0}$ (Tabela 3). Já a $\mathrm{CS}_{100}$ permite minimizar significativamente $(\mathrm{p}<0,005)$ não só as perdas de solo, bem como as de matéria orgânica e de nutrientes em relação a $\mathrm{CS}_{0}$ e $\mathrm{CS}_{50}$. Deve-se considerar que a não diferença nas concentrações de $\mathrm{K}$ no sedimento, para os tratamentos $\mathrm{CS}_{0}, \mathrm{CS}_{50}$ e $\mathrm{CS}_{100}$, pode ser devida ao manejo da área, que se caracteriza pela constante aplicação de vinhaça em área total.

TABELA 4. Concentração média de matéria orgânica e nutrientes no sedimento erodido em função da cobertura do solo. Organic matter and nutrients contents in eroded sediment under different soil cover.

\begin{tabular}{|c|c|c|c|c|c|}
\hline $\begin{array}{c}\text { Cobertura do solo } \\
\%\end{array}$ & $\begin{array}{c}\mathrm{MO} \\
\mathrm{g} \mathrm{dm}^{-3}\end{array}$ & $\begin{array}{c}\mathrm{P} \\
\mathrm{mg} \mathrm{dm}^{-3}\end{array}$ & K & $\begin{array}{c}\mathrm{Ca} \\
\text { nmolc di }\end{array}$ & $\mathrm{Mg}$ \\
\hline 0 & $43,7 \mathrm{a}$ & $128,7 \mathrm{a}$ & $2,6 \mathrm{a}$ & $78,0 \mathrm{a}$ & $26,3 \mathrm{a}$ \\
\hline 50 & $30,7 \mathrm{a}$ & $91,0 \mathrm{a}$ & $3,3 \mathrm{a}$ & $71,6 \mathrm{a}$ & $23,3 \mathrm{a}$ \\
\hline 100 & $13,7 \mathrm{~b}$ & $15,3 \mathrm{~b}$ & $2,0 \mathrm{a}$ & $20,3 \mathrm{~b}$ & $6,6 \mathrm{~b}$ \\
\hline
\end{tabular}

Médias seguidas de mesma letra na coluna não diferem significativamente, pelo teste Tukey, a 5\% de probabilidade.

$\mathrm{Na}$ Tabela 5, é apresentada a taxa de enriquecimento do sedimento erodido (ER) por MO e nutrientes $(\mathrm{P}, \mathrm{K}, \mathrm{Ca}$ e $\mathrm{Mg})$. A ER é uma relação entre a concentração de $\mathrm{MO}$ e nutrientes no sedimento erodido e a no solo original. Quando a ER é maior que 1, significa que o sedimento se encontra enriquecido por matéria orgânica ou nutriente do solo (HERNANI et al., 1999; SILVA et al., 2005). Logo, em quase todas as condições testadas, houve enriquecimento do sedimento em relação ao solo original (Tabela 5). Tal constatação, segundo SILVA et al. (2005), caracteriza a seletividade do material transportado durante o processo de erosão, o qual é constituído principalmente por partículas mais finas, as frações mais reativas do solo, e, consequentemente, com maior capacidade de carrear matéria orgânica e nutrientes.

TABELA 5. Taxa de enriquecimento do sedimento (ER) por matéria orgânica (MO) e nutrientes. Enrichment ratios (ER) of organic matter (MO) and nutrients.

\begin{tabular}{cccccc}
\hline \multirow{2}{*}{ Cobertura do Solo (CS \%) } & \multicolumn{5}{c}{ Taxa de Enriquecimento (ER) } \\
\cline { 2 - 6 } & $\mathrm{MO}$ & $\mathrm{P}$ & $\mathrm{K}$ & $\mathrm{Ca}$ & $\mathrm{Mg}$ \\
\hline 0 & $2,7 \mathrm{a}$ & $3,8 \mathrm{a}$ & $1,3 \mathrm{a}$ & $3,9 \mathrm{a}$ & $2,9 \mathrm{a}$ \\
50 & $1,9 \mathrm{a}$ & $2,7 \mathrm{ab}$ & $1,7 \mathrm{a}$ & $3,6 \mathrm{a}$ & $2,6 \mathrm{a}$ \\
100 & $0,9 \mathrm{~b}$ & $0,5 \mathrm{~b}$ & $1,0 \mathrm{a}$ & $1,0 \mathrm{~b}$ & $0,7 \mathrm{~b}$ \\
\hline
\end{tabular}

Médias seguidas de mesma letra na coluna não diferem significativamente, pelo teste Tukey, a 5\% de probabilidade. 
Num Latossolo Vermelho (LV) com declive de $0,08 \mathrm{~m} \mathrm{~m}^{-1}$, sob cultivo de cana-de-açúcar, com despalha obtida com o uso do fogo, IZIDORIO et al. (2005) determinaram, com o solo descoberto, taxas de enriquecimento por $\mathrm{MO}, \mathrm{P}, \mathrm{K}, \mathrm{Ca}$ e $\mathrm{Mg}$ da ordem de 1,$62 ; 3,87 ; 0,73 ; 1,27$ e 1,24, respectivamente. No presente trabalho, as taxas de ER foram 2,7 (MO), 3,8 (P), 1,3 (K), 3,9 $(\mathrm{Ca})$ e 2,9 $(\mathrm{Mg})$ para $\mathrm{CS}_{0}$ (Tabela 3). Verifica-se, portanto, que as taxas de ER no Argissolo estudado foram superiores àquelas obtidas num LV por IZIDORIO et al. (2005). As diferenças entre os valores das taxas de enriquecimento apresentadas na Tabela 3 em relação aos valores encontrados por IZIDORIO et al. (2005), provavelmente, são devidas ao fato de que a presença de nutrientes nos sedimentos varia conforme sua concentração no solo, o que tem influência direta das adubações e da cobertura e manejo do solo, como demonstrado em diversos trabalhos (BERTOL et al., 2004; BERTOL et al., 2007). Tais diferenças podem ainda ser devidas à maior erodibilidade do Argissolo em relação ao LV utilizado por IZIDORIO et al. (2005). Essa suposição parece fazer sentido, uma vez que o efeito do relevo (LS) no processo de erosão foi maior no caso de IZIDORIO et al. (2005), ou seja, LS igual a 0,595 contra 0,337 do Argissolo em questão.

AMARAL (2003) determinou para o Argissolo estudado o valor de erodibilidade (Fator K) igual a $0,023 \mathrm{Mg}$ ha h ha ${ }^{-1} \mathrm{MJ}^{-1} \mathrm{~mm}^{-1}$. Contudo, a erodibilidade (fator $\mathrm{K}$ ) determinada no presente trabalho foi de $0,039 \mathrm{Mg}$ ha h ha ${ }^{-1} \mathrm{MJ}^{-1} \mathrm{~mm}^{-1}$, cujo valor é considerado alto (SILVA \& ALVARES, 2005). Já IZIDORIO et al. (2005) determinaram para o Latossolo Vermelho (LV), por ele utilizado, um fator $\mathrm{K}$ igual a $0,010 \mathrm{Mg}$ ha $\mathrm{h} \mathrm{ha}^{-1} \mathrm{MJ}^{-1} \mathrm{~mm}^{-1}$, erodibilidade baixa (SILVA \& ALVARES, 2005). Os valores das concentrações de MO, P, K, Ca e Mg no LV, obtidos por IZIDORIO et al. (2005), foram de $28 \mathrm{~g} \mathrm{dm}^{-3}, 26 \mathrm{mg} \mathrm{dm}^{-3}, 5 \mathrm{mmol}_{\mathrm{c}} \mathrm{dm}^{-3}, 38 \mathrm{mmol}_{\mathrm{c}} \mathrm{dm}^{-3}$ e $13 \mathrm{mmol}_{\mathrm{c}} \mathrm{dm}^{-3}$, respectivamente. Essas concentrações iniciais no LV são todas superiores às obtidas no Argissolo deste trabalho, na profundidade de 0,00-0,20 m, exceto para fósforo (Tabela 1). Logo, além da concentração inicial de nutrientes no solo original, a maior suscetibilidade à erosão também deve ter influenciado numa maior taxa de enriquecimento do sedimento do Argissolo.

Pelos resultados apresentados, não ocorreu enriquecimento significativo $(\mathrm{p}>0,05)$ do sedimento para $\mathrm{CS}_{100}$ (Tabela 5), o que demonstra a eficiência da palha de cana-de-açúcar na redução da concentração de matéria orgânica e nutrientes no material erodido. Segundo NEARING et al. (2001), considerável enriquecimento pode ocorrer durante os processos de transporte e deposição. Essa última observação justifica-se pelos resultados obtidos nas parcelas com $\mathrm{CS}_{0} \mathrm{e}$ $\mathrm{CS}_{50}$, cujos controles no escoamento superficial foram, em termos percentuais, em ordem, zero e $18 \%$, como já destacado. Nessas parcelas, as taxas de enriquecimento por $\mathrm{MO}, \mathrm{P}, \mathrm{K}, \mathrm{Ca}$ e $\mathrm{Mg}$ sempre foram superiores às determinadas com $\mathrm{CS}_{100}$.

É possível justificar, pelos resultados obtidos neste trabalho, que quaisquer efeitos que reduzam significativamente as concentrações de $\mathrm{MO}$ e de nutrientes no sedimento erodido só ocorrem quando a percentagem de resíduos em contato com a superfície do Argissolo é superior a $50 \%$.

As perdas de $\mathrm{MO}$ e nutrientes por erosão são apresentadas na Tabela 6. Os resultados apresentados são concordantes com CASTRO et al. (1986), os quais observaram que as perdas de matéria orgânica e nutrientes têm significativa correlação com as perdas de solo. Observando-se os resultados apresentados na Tabela $3 \mathrm{e}$, ainda, os da Tabela 6 , fica evidente que as maiores e significativas perdas de solo, $\mathrm{MO}$ e nutrientes ocorreram no tratamento $\mathrm{CS}_{0}$.

Os resultados aqui apresentados demonstram que técnicas que privilegiam o manejo dos resíduos, principalmente pela manutenção desses sobre a superfície do solo, efetivamente controlam a erosão hídrica como mencionam THOMPSON et al. (2001) e BEZERRA \& CANTALICE (2006). Portanto, o sistema de colheita mecanizada da cana-de-açúcar, que permite a manutenção da palhada sobre a superfície do solo, reduz as perdas de MO e de nutrientes no sedimento erodido. Contudo, as perdas de nutrientes e/ou produtos químicos com a água da enxurrada podem ser elevadas em sistemas conservacionistas (THOMPSON et al., 2001). Tal fato ocorrerá se os corretivos e fertilizantes forem aplicados em superfície e não incorporados ao solo, principalmente 
se ocorrerem enxurradas dias após a aplicação daqueles produtos. Isso poderá ser um problema crítico para a qualidade das águas superficiais, segundo THOMPSON et al. (2001). Não foram feitas avaliações dos nutrientes contidos na água da enxurrada neste trabalho. Logo, fica sugestão para que isso seja avaliado, num futuro próximo, em áreas com colheita de cana crua.

TABELA 6. Perdas de matéria orgânica (MO) e nutrientes por erosão. Organic matter and nutrients losses by soil erosion.

\begin{tabular}{crcccr}
\hline \multirow{2}{*}{ Cobertura do Solo $(\mathrm{CS} \%)$} & \multicolumn{5}{c}{ Perdas por Erosão $\left(\mathrm{kg} \mathrm{ha}^{-1}\right)$} \\
\cline { 2 - 6 } & $\mathrm{MO}$ & $\mathrm{P}$ & $\mathrm{K}$ & $\mathrm{Ca}$ & $\mathrm{Mg}$ \\
\hline 0 & $172,6 \mathrm{a}$ & $0,44 \mathrm{a}$ & $0,42 \mathrm{a}$ & $6,33 \mathrm{a}$ & $1,27 \mathrm{a}$ \\
50 & $40,7 \mathrm{~b}$ & $0,09 \mathrm{ab}$ & $0,17 \mathrm{~b}$ & $1,96 \mathrm{~b}$ & $0,39 \mathrm{~b}$ \\
100 & $15,9 \mathrm{c}$ & $0,02 \mathrm{~b}$ & $0,08 \mathrm{~b}$ & $0,52 \mathrm{~b}$ & $0,10 \mathrm{~b}$ \\
\hline
\end{tabular}

Médias seguidas de mesma letra na coluna não diferem significativamente, pelo teste Tukey, a 5\% de probabilidade.

\section{CONCLUSÕES}

As taxas de infiltração de água no solo são maiores nas áreas com 50\% e 100\% de cobertura por resíduos vegetais e, nessa ordem, a erosão é reduzida em até $68 \%$ e $89 \%$ em relação ao solo.

As concentrações médias no sedimento erodido dos nutrientes são significativamente reduzidas quando o solo apresenta $100 \%$ de cobertura por resíduos vegetais de cana-de-açúcar.

Considerável enriquecimento do sedimento por MO e nutrientes ocorre, se a cobertura por resíduos de cana-de-açúcar sobre a superfície do solo for igual ou inferior a 50\%.

\section{REFERÊNCIAS}

AMARAL, N.S. do. Variabilidade espacial da expectativa e risco de erosão num Argissolo sob cultivo de cana-de-açúcar em Catanduva - SP. 2003. 74f. Monografia (Trabalho de Graduação) Faculdade de Ciências Agrárias e Veterinárias, Universidade Estadual Paulista, Jaboticabal.

BERTOL, I.; ENGEL, F.L.; MAFRA, A.L.; BERTOL, O.J.; RITTER, S.R. Phosphorus, potassium and organic carbon concentrations in runoff water and sediments under different soil tillage systems during soybean growth. Soil \& Tillage Research, Amsterdam, v.94, n.1, p.142-150, 2007.

BERTOL, I.; GUADAGNIN, J.C.; CASSOL, P.C.; AMARAL, A.J.; BARBOSA, F.T. Perdas de fósforo e potássio por erosão hídrica em um inceptisol sob chuva natural. Revista Brasileira de Ciência do Solo, Viçosa, v.28, n.3, 485-494, 2004.

BEZERRA, S.A.; CANTALICE, J.R.B. Erosão entressulcos em diferentes condições de cobertura vegetal de solo, sob cultivo da cana-de-açúcar. Revista Brasileira de Ciência do Solo, Viçosa, v.30, n.3, p. 565-573, 2006.

BRADY, N.C.; WEIL, R.R. The nature and properties of soils. $13^{\text {th }}$ ed. New Jersey: Prentice Hall, 2002. $960 \mathrm{p}$.

BRAIDA, J.A.; CASSOL, E.A. Relações da erosão em entressulcos com o tipo e quantidade de resíduo vegetal na superfície do solo. Revista Brasileira de Ciência do Solo, Viçosa, v.23, n.3, 711721, 1999.

CASSOL, E.A.; CANTALICE, J.R.B.; REICHERT, J.M.; MONDARDO, A. Escoamento superficial e desagregação do solo em entressulcos em solo franco-argilo-arenoso com resíduos vegetais. Pesquisa Agropecuária Brasileira, Brasília, v.39, n.7, p.685-690, 2004.

CASSOL, E.A.; LIMA, V.S. Erosão em entressulcos sob diferentes tipos de preparo e manejo do solo. Pesquisa Agropecuária Brasileira, Brasília, v.38, n.1, p.117-124, 2003. 
CASTRO, O.M.; LOMBARDI NETO, F.; QUAGGIO, J.A.; MARIA, I.C.; VIEIRA,S.R.; DECHEN, S.C.F. Perdas por erosão de nutrientes vegetais na sucessão soja/trigo em diferentes sistemas de manejo. Revista Brasileira de Ciência do Solo, Viçosa, v.10, n.3, p.293-297, 1986.

EMPRESA BRASILEIRA DE PESQUISA AGROPECUÁRIA. Manual de métodos de análise de solo. 2.ed. Rio de Janeiro, 1997. 212 p.

EMPRESA BRASILEIRA DE PESQUISA AGROPECUÁRIA. Centro Nacional de Pesquisa de Solos. Sistema brasileiro de classificação de solos. Brasília, 1999. 412 p.

FOSTER, G.R. Modeling the erosion process. In: HAAN, C.T.; JOHNSON, H.P.; BRAKENSSIEK, D.L. Hidrologic modeling of small watersheds. St Joseph: American Society of Agricultural Engineers, 1982. p.296-380. (Monograph, 5).

HERNANI, L.C.; KURIHARA, C.H.; SILVA W.M. Sistema de manejo de solos e perdas de nutrientes e matéria orgânica por erosão. Revista Brasileira de Ciência do Solo, Viçosa, v.23, n.1, p.145-154, 1999.

IZIDORIO, R. Perdas de nutrientes e sua distribuição espacial num Latossolo Vermelho. 2004. 68f. Dissertação (Mestrado em Ciência do Solo) - Faculdade de Ciências Agrárias e Veterinárias, Universidade Estadual Paulista, Jaboticabal, 2004.

IZIDORIO, R.; MARTINS FILHO, M.V.; MARQUES JÚNIOR, J. ; SOUZA, Z.M.; PEREIRA, G.T. Perdas de nutrientes por erosão e sua distribuição espacial em área sob cana-de-açúcar. Engenharia Agrícola, Jaboticabal, v.25, n.3, p.660-670, 2005.

LAFLEN, J.M.; FOSTER, G.R.; ONSTAD, C. Simulation of individual storm soil losses for modeling the impact of soil erosion on cropland productivity. In: EL-SWAFY, S.A.;

MULDENHAUER. W.C.; ANDDREW, L. (Ed.). Soil erosion and conservation. Ankeny: SCSA, 1985. p.285-295.

MARTINS FILHO, M.V. Influência da compactação do solo nas perdas por erosão e na sua erodibilidade. 1987. 95 f. Monografia (Trabalho de Graduação) - Faculdade de Ciências Agrárias e Veterinárias, Universidade Estadual Paulista, Jaboticabal, 1987.

MARTINS FILHO, M.V. Modelagem do processo de erosão entressulcos em latossolos de Jaboticabal - SP. 1999. 140 f. Tese (Doutorado em Solos e Nutrição de Plantas) - Universidade Federal de Lavras, Lavras, 1999.

MARTINS FILHO, M.V.; CAMPOS, M.P.; IZIDORIO, R.; COTRIN, F.B.; SERRA, E.A.; AMARAL, N.S.; SOUZA, Z. Modelos para estimativas do subfator cobertura-manejo relativo à erosão entressulcos. Engenharia Agrícola, Jaboticabal, v.24, n.3, p.603-611, 2004.

NEARING, M.A.; NORTON, D.L; ZHANG, X Soil erosion and sedimentation. In: RITTER, W.F.; SHIRMOHAMMADI, A. (Eds.). Agricultural nopoint source pollution. Boca Raton: Lewis Publishers, 2001. p.29-58.

RAIJ, B. van; QUAGGIO, J.A.; CANTARELLA, H.; FERREIRA, M.E.; LOPES, A.S.; BATAGLIA, O.C. Análise química do solo para fins de fertilidade. Campinas: Fundação Cargill, 1987. $170 \mathrm{p}$.

SCHAEFER, C.E.G.R.; SILVA, D.D.; PAIVA, K.W.N.; PRUSKI, F.F.; ALBUQUERQUE FILHO, M.R.; ALBUQUERQUE, M.A. Perdas de solo, nutrientes, matéria orgânica e efeitos microestruturais em Argissolo Vermelho-Amarelo sob chuva simulada. Pesquisa Agropecuária Brasileira, Brasília, v.37, n.5, p. 669-678, 2002.

SHELTON, D.P.; KANABLE, R,; JASA, P.J. Estimating percent residue cover using the linetransect method. 1993. NebGuide G93-1133, University of Nebraska, Lincoln, 4 p. Disponível em: http://www.ianr.unl.edu/pubs/fieldcrops/g1133.htm. Acesso em: 7 jan. 2005. 
SILVA, A.M.; ALVARES, C.A. Levantamento de informações e estruturação de um banco de dados sobre a erodibilidade de classes de solos no Estado de São Paulo. Geociências, Rio Claro, v.24, n.1, p.33-42, 2005.

SILVA, A.M.; SILVA, M.L.N.; CURI, N.; LIMA, J.M.; AVANZI, J.C.; FERREIRA, M.M. Perdas de solo, água, nutrientes e carbono orgânico em Cambissolo e Latossolo sob chuva natural. Pesquisa Agropecuária Brasileira, Brasília, v.40, n.12, p.1.223-1.230, 2005.

STATSOFT. Statistica: quick reference. Tulsa, 1994. 148 p.

THOMPSON, A.L.; GHIDEY, F.; REDMI, T.P. Raindrop energy effects on chemical and sediment transport. Transactions of the ASAE, St Joseph, v.44, n.4, p.835-841, 2001.

WISCHMEIER, W.H. Estimating the soil loss equations cover and management factor for undisturbed areas. In: Present and prospective technology for predicting sediment yields and sources. Washington: Agricultural Research, 1975. p.118-124. (ARS-S-40)

WISCHMEIER, W.H.; SMITH, D.D. Predicting rainfall erosion losses; a guide to conservation planning. Washington: Department of Agriculture, 1978. 58 p. (Agriculture Handbook, 537) 